\title{
Comparative evaluation of the shear bond strength of ceramic brackets of three different base designs bonded to amalgam and composite restorations with different surface treatment
}

\author{
Maryam Shirazi ${ }^{1, A, D-F}$, Monirsadat Mirzadeh ${ }^{2, C, E, F}$, Malek Modirrousta ${ }^{3, B, D, F}$, Sepideh Arab ${ }^{4, C, F}$ \\ 1 Private dental office, Tehran, Iran \\ ${ }^{2}$ Metabolic Diseases Research Center, Qazvin University of Medical Sciences, Iran \\ ${ }^{3}$ Student Research Committee, Qazvin University of Medical Sciences, Iran \\ ${ }^{4}$ School of Dentistry, Tehran University of Medical Sciences, Iran \\ A - research concept and design; $B$ - collection and/or assembly of data; $C$ - data analysis and interpretation; \\ $\mathrm{D}$ - writing the article; $\mathrm{E}$ - critical revision of the article; $\mathrm{F}$ - final approval of the article
}

Address for correspondence

Maryam Shirazi

E-mail:dr.maryam_shirazi@yahoo.com

Funding sources

None declared

Conflict of interest

None declared

Received on December 9, 2019

Reviewed on February 24, 2020

Accepted on December 15, 2020

Published online on March 31, 2021

Cite as

Shirazi M, Mirzadeh M, Modirrousta M, Arab S. Comparative evaluation of the shear bond strength of ceramic brackets of three different base designs bonded to amalgam and composite restorations with different surface treatment. Dent Med Probl. 2021;58(2):193-200.

doi:10.17219/dmp/131684

DOI

$10.17219 / \mathrm{dmp} / 131684$

\section{Copyright}

○) 2021 by Wroclaw Medical University

This is an article distributed under the terms of the

Creative Commons Attribution 3.0 Unported License (CC BY 3.0)

(https://creativecommons.org/licenses/by/3.0/).

\begin{abstract}
Background. Nowadays, due to an increasing number of adult orthodontic applicants, who also have multiple dental restorations, it is important to have the ability to bond an orthodontic appliance to restoration surfaces.

Objectives. The aim of this paper was to determine the shear bond strength of ceramic brackets of 3 different base designs bonded to amalgam and composite restorations after using different surface treatment methods in vitro.

Material and methods. In an in vitro study, the surfaces of 180 amalgam and composite specimens were prepared by using sandblasting and the erbium, chromium-doped yttrium, scandium, gallium, and garnet (Er,Cr:YSGG) laser irradiation. Ceramic brackets of 3 base designs, including Star, Cross and Slot, for upper central teeth were bonded to amalgam and composite surfaces by using Transbond ${ }^{\mathrm{TM}}$ XT Light Cure Adhesive. All specimens were incubated at a temperature of $37^{\circ} \mathrm{C}$ for 1 week, and then subjected to shear bond strength tests after 1,000 cycles of thermal cycling. The bond strength of the groups was analyzed by means of the one-way and two-way analysis of variance (ANOVA), and the comparison of the 2 groups was made with Tukey's test. Residual adhesives were also determined by means of a 4-part criterion (0-3) in different groups and the results were analyzed with the $x^{2}$ test.

Results. There was a significant difference in the shear bond strength of brackets of 3 base designs bonded to amalgam and composite surfaces with different surface treatment.

Conclusions. According to the results of this study, all surface treatment methods and different bracket base designs were able to provide sufficient shear bond strength on composite and amalgam surfaces. As far as the bracket base design is concerned, the use of each of the 3 base designs in the amalgam group brought desirable effects in terms of the adhesive remnant index (ARI) and shear bond strength values; the use of the Star base design in the composite group proved to be suitable.
\end{abstract}

Keywords: shear bond strength, orthodontic brackets, adhesive remnant index 


\section{Introduction}

In contemporary orthodontic treatment, establishing a successful clinical bond between the bracket and the tooth surface is essential. Currently, the number of patients applying for orthodontic treatment is on the rise, and adults' treatment has also grown rapidly. Orthodontic treatment for both clinical and cosmetic purposes has long been popular among teenagers, but in recent times, middle-aged patients and seniors have also sought orthodontic treatment more frequently than previously. These patients commonly received amalgam or metal restorations in their earlier dental treatment. At least 1 amalgam restoration was found in $50-85 \%$ of the population. ${ }^{1} \mathrm{Re}-$ cent orthodontic treatment requires bonding orthodontic appliances to restoration surfaces, including resin, amalgam and porcelain composites, and thus achieving a reliable bond to restoration surfaces is important. As adults tend to look esthetic, they use ceramic brackets and have dental restorations, many studies have been conducted on the bond strength of orthodontic brackets on restoration surfaces, such as amalgam or porcelain. It should be high enough to maintain orthodontic brackets during orthodontic treatment ${ }^{2}$ and to withstand the forces caused by occlusion, chewing and wire stressing as well as biomechanical forces. On the other hand, bond strength must not be too high so that to avoid causing damage to the tooth surface during debonding at the end of the treatment. $^{3}$

To be accepted, a bracket bond system must be able to withstand the forces of orthodontic wires as well as those of the oral environment. Bond strength testing is one of the most popular analyses used in dental material evaluation. The force used in these tests is often of tensile or shear type. In a comparison between tensile, shear and torsional forces, it has been identified that shear forces are the most common and destructive type of forces, which cause bracket debonding in the mouth. ${ }^{4,5}$

Things get even tougher when we want to bond brackets on dental restorations, and not on the enamel. Various surface preparation methods as well as different kinds of physical and chemical retention are used to do this. ${ }^{6}$

Some research has been carried out on the effects of various surface preparation methods, such as diamond milling, sandblasting, or etching with phosphoric acid and hydrofluoric acid. ${ }^{7}$ Sandblasting or aerating is a method of combining aluminum oxide particles with air pressure to increase bond strength to gold, porcelain, amalgam, and composite resins. Other clinical applications of sandblasting include removing the composite from the surface of the debonded bracket, increasing the retention of orthodontic bands and making the surface of primary teeth rough. ${ }^{8}$ Sandblasting is the most common way of surface preparation and increases bond strength by creating scratch-like irregularities. ${ }^{9}$
Recently, the Er,Cr:YSGG laser system has also been considered for surface preparation, and it has been determined that this type of laser produces a rough surface on the enamel and the dentin, similarly to the conventional acid-etching technique. ${ }^{9}$ Limited studies have evaluated the effects of laser irradiation on the surface of metal restorations and it has been found that Er,Cr:YSGG lasers can partially cause abrasion on amalgam surfaces. ${ }^{10}$

Ceramic brackets are a type of orthodontic brackets made of non-metal minerals that are almost colorless. This type of brackets is used for people who want the orthodontic appliances on their teeth to be almost unnoticeable, not attracting the attention of others. Currently, all the available ceramic brackets are made of aluminum oxide. These brackets are divided into 2 types at the manufacturing stage - polycrystalline and monocrystalline, and the main difference is in the transparency of the brackets; single-crystal brackets are more transparent than polycrystalline ones. Fortunately, both single-crystal and polycrystalline brackets are resistant to staining and discoloration. ${ }^{11}$

Mechanical bonding is caused by the clogged spots and surface roughness, and the locking of the materials. The amount of the created microscopic retention indicates the strength of the bond. Almost all dental bonding consists in mechanical bonding. Chemical bonding is also possible, but this type of bond often accounts for a small share of the total bond strength. ${ }^{12}$ For mechanical bonding, various bracket base designs, such as microcrystalline, mechanical, button, and polymer bases are available. ${ }^{13}$ Different bracket base designs are used to achieve proper mechanical retention and bonding of orthodontic appliances to different dental surfaces or restorative materials; it has been shown that different bracket base designs show different bond strength. ${ }^{14,15}$

Bracket failure is divided into 2 groups with regard to its location: cohesive failure, which occurs inside the adhesive; and adhesive failure, which occurs at the site of the tooth-adhesive or bracket-adhesive interface.

The adhesive remnant index (ARI) is used to classify the bond failure location. ${ }^{2}$ Based on the material outlined and the availability of 3 different base designs (Star base, Cross base and Slot base), we intended to use both the sandblasting and laser surface treatment methods, due to their frequent application, to examine the bond strength of ceramic brackets of these 3 different base designs on amalgam and composite restorations.

\section{Material and methods}

This in vitro study was conducted on 180 samples of amalgam and composite in 12 groups of 15 (for each of the 3 bracket base designs, each kind of surface - amalgam or composite, and each method of surface preparation - sandblasting or laser). There were 3 groups of 60 
for each bracket base design; half of the specimens were bonded to amalgam surfaces and the other half to composite surfaces. The grouping was done randomly.

To simulate the amalgam restoration cavities, 2 grooves, $3.5 \mathrm{~mm}$ in diameter and $6 \mathrm{~mm}$ in width, with smooth baffles and parallel walls perpendicular to the surface, were cut on brass ingots. This was accomplished by using a milling machine and a cylindrical turning machine. For composite samples, 90 Filtek $^{\circledR}$ Z250 composite discs (3M ESPE, St. Paul, USA) of color A2, a 10-millimeter diameter and a 2-millimeter thickness were prepared by the operator and, using the Ortholux ${ }^{\circledR}$ LED device (3M Unitek, Monrovia, USA) with radiometer adequacy, they were illuminated at a moderate intensity for $40 \mathrm{~s}$ on both sides. ${ }^{16}$ The head was at a $90^{\circ}$ angle to the surface of the discs. They were then evaluated ophthalmologically for the absence of cracks or any defects.

In the sandblasting group, amalgam and composite surfaces were sandblasted with the DENTO-PREPTM microbeader (Rønvig Dental, Daugård, Denmark) using 50-micrometer aluminum oxide particles, at air pressure of $3 \mathrm{~kg} / \mathrm{cm}^{2}$ at a distance of $10 \mathrm{~mm}$ for $4 \mathrm{~s}$. A plaster facade after washing and drying amalgam and composite surfaces was considered as a criterion for finishing the sandblasting operation. ${ }^{17}$

In the laser group, an Er,Cr:YSGG laser machine (Biolase Europe, Floss, Germany) with a G-type 600-micron tip was used to prepare the surface. This laser system produces photons with a wavelength of $2.78 \mu \mathrm{m}$, with a duration of $140-200 \mu \mathrm{m}$ and a repetition rate of $20 \mathrm{~Hz}$. The output power varies from 0 to $6 \mathrm{~W}$. In this study, a power of $1 \mathrm{~W}$ (20\% air level and $10 \%$ water level) was used. The rays were irradiated perpendicular to the surface at a distance of $1 \mathrm{~mm}$ with a radiation time of $5 \mathrm{~s}$. The droplet size was $0.228 \mathrm{~mm}$ and the energy density of the laser was $17.7 \mathrm{~J} / \mathrm{cm}$. After irradiation, amalgam and composite samples were rinsed with distilled water and finally dried. ${ }^{5}$

Upper central incisor ceramic brackets (0.022-inch slot size) and a base of a surface area of $12.25 \mathrm{~mm}^{2}$ were bonded to the center of the amalgam and composite surfaces of the specimens from 12 groups. The Transbond ${ }^{\circledR} \mathrm{XT}$ bonding agent and Transbond XT Light Cure Adhesive (3M Unitek) containing $45-55 \%$ of bisphenol A diglycidyl methacrylate (Bis-GMA) and 45-55\% of triethylene glycol dimethacrylate (TEGDMA) were used for bonding the attachments. The Transbond XT bonding agent was applied to amalgam and composite surfaces with the help of a microbrush, dried with a slight pressure of air spray (no water or oil) and left to dry for $10 \mathrm{~s}$. Transbond XT Light Cure Adhesive was applied on the back of the bracket, and then the bracket was compressed on amalgam and composite surfaces with the use of an orthodontic gage. Excess composite was removed by using the sharp tip of an explorer. After making sure that the desired condition was reached, the curing operation of the composite was carried out with a medium-intensity light-curing apparatus, ${ }^{8}$ in which the light was spun for $40 \mathrm{~s}$ perpendicular to the surface of the ceramic bracket face. ${ }^{18}$

All samples were stored in an incubator (Pars Azma Co., Tehran, Iran) at $37^{\circ} \mathrm{C}$ and $95 \%$ humidity for 1 week, and then thermocycled for 10,000 rounds, using a thermal cycler (Dorsa, Tehran, Iran). They were placed in $5^{\circ} \mathrm{C}$ in cold water for $20 \mathrm{~s}$ and in $55^{\circ} \mathrm{C}$ in warm water for $20 \mathrm{~s}$ in each cycle, and were kept for $5 \mathrm{~s}$ out of the water container, between the 2 containers. ${ }^{10}$ Due to the fact that orthodontic adhesives are routinely exposed to thermal changes in the oral cavity, it is paramount to establish whether these changes introduce stress in the adhesive that might affect bond strength. Thermal cycling is an in vitro process through which the adhesive resin is subjected to temperature extremes, compatible with the oral cavity environment. ${ }^{19}$

In order to mount the samples in acrylic resin for bond strength testing, wax boxes were fabricated and the brackets were placed on top of the boxes ( 5 per each row) with $0.019 \times 0.025$-inch wire ratchets, using elastomeric ligatures in such a way that they were parallel to the longitudinal margins of the box. Autopolymerizing acrylic resin was poured into the box up to the upper margin of composite discs. The samples were embedded in the acrylic resin. The contact of the acrylic resin and the bracket was prevented as such, and a proper stub was obtained for the placement of the samples in a universal testing machine. ${ }^{16}$ To measure shear bond strength, the samples were placed in a universal testing machine (STM-20; SANTAM Engineering Design Co. Ltd., Tehran, Iran) with pooling tools of a 0.02-inch ligature wire. The ligature wire was modified and connected with the bracket as a shear tool, and a direct force was applied at a speed of $1 \mathrm{~mm} / \mathrm{min}$ until the bracket fell apart. The load at fracture was recorded in newtons. By dividing the load $[\mathrm{N}]$ by the cross-sectional area of the bracket $\left[\mathrm{mm}^{2}\right]$, the shear bond strength of the brackets was calculated [MPa]. The samples were evaluated under a stereomicroscope (Carl Zeiss, Jena, Germany) at $\times 10$ magnification to determine the mode of failure. The ARI score was also calculated based on the amount of adhesive remaining on the surface, using a 4-point scale as follows ${ }^{3}$ :

- score 0: no adhesive remained on the restoration surface;

- score 1: less than $50 \%$ of the adhesive remained on the restoration surface;

- score 2: more than $50 \%$ of the adhesive remained on the restoration surface;

- score 3: all the adhesive remained on the restoration surface.

The IBM SPSS Statistics for Windows software, v. 20, was used for data analysis. For this purpose, the mean $(M)$ and standard deviation $(S D)$ for the shear bond strength values for 12 sample groups were calculated and reported. 
The data was analyzed by means of the one-way and twoway analysis of variance (ANOVA), and Tukey's test for paired comparisons. Also, the ARI scores after debonding were compared among the groups with the $X^{2}$ test. A $p$-value $<0.05$ was considered statistically significant.

\section{Results}

In this study, according to the one-way ANOVA, significant differences were noted in shear bond strength between the 12 experimental groups.

The comparison of shear bond strength in the amalgam vs composite groups, sandblasting vs laser groups and 3 bracket base design groups revealed significant differences (Table 1).

The comparison of shear bond strength in the composite group with sandblasting and laser surface treatment revealed no significant difference, but in the amalgam group with sandblasting and laser surface treatment, there was a significant difference (Table 2).

The comparison of shear bond strength in the composite group with 3 bracket base designs revealed a significant difference, but in the amalgam group with 3 bracket base designs, there was no significant difference (Table 3).

\section{Adhesive remnant index}

According to the results shown in Table 4, the difference in the ARI scores between the composite and amalgam groups was significant at the 0.05 level of probability.
Table 2. Distribution and comparison of the mean shear bond strength $[\mathrm{MPa}]$ in the composite and amalgam groups with sandblasting and laser surface preparation

\begin{tabular}{|c|c|c|c|c|}
\hline $\begin{array}{c}\text { Restorative } \\
\text { material }\end{array}$ & $\begin{array}{c}\text { Surface } \\
\text { preparation }\end{array}$ & M & SD & $p$-value \\
\hline \multirow{2}{*}{ Composite } & sandblasting & 9.22 & 5.32 & \\
& laser & 10.15 & 5.15 & 0.400 \\
Amalgam & sandblasting & 8.46 & 2.48 & \\
& laser & 4.64 & 1.47 & $0.001^{*}$ \\
\hline
\end{tabular}

* statistically significant.

Most composite specimens (71.1\%) had an ARI score of 3 ( $100 \%$ of the adhesive remained on the restoration) and most amalgam samples (88.8\%) showed an ARI score of 0 (no adhesion remained on the restoration). The highest fracture incidence in the composite base was observed in the case of the Cross base design of the composite groups. In the composite + laser + Star group, fractures in the composite base were observed in 2 samples.

\section{Discussion}

One of the difficulties in fixed orthodontic treatment is peeling off the brackets from the tooth surface. In other words, the repeated removal of orthodontic attachments is a major problem in orthodontics. On the other hand, with an increasing number of adults with multiple restorations, requesting orthodontic treatment and with regard to the interest of these patients in ceramic brackets,

Table 1. Distribution and comparison of the mean shear bond strength [MPa] in different groups

\begin{tabular}{|c|c|c|c|c|c|c|}
\hline \multicolumn{2}{|c|}{ Groups } & $M$ & $S D$ & Min & Max & $p$-value \\
\hline \multirow{2}{*}{ Restorative material } & composite & 9.68 & 2.44 & 2.77 & 22.06 & \multirow{2}{*}{$0.001^{*}$} \\
\hline & amalgam & 6.55 & 1.61 & 1.89 & 14.22 & \\
\hline \multirow{2}{*}{ Surface preparation } & sandblasting & 8.84 & 2.06 & 2.77 & 22.06 & \multirow{2}{*}{$0.030^{*}$} \\
\hline & laser & 7.40 & 1.99 & 1.89 & 19.53 & \\
\hline \multirow{3}{*}{ Bracket base design } & Star & 8.84 & 2.46 & 3.58 & 13.71 & \multirow{3}{*}{$0.001^{*}$} \\
\hline & Cross & 11.46 & 5.47 & 1.89 & 22.60 & \\
\hline & Slot & 5.29 & 2.18 & 2.60 & 12.51 & \\
\hline
\end{tabular}

M - mean; SD - standard deviation; min - minimum; max - maximum; ${ }^{*}$ statistically significant.

Table 3. Distribution and comparison of the mean shear bond strength [MPa] in the composite and amalgam groups with different bracket base designs

\begin{tabular}{|c|c|c|c|c|c|c|}
\hline Restorative material & Bracket base design & M & $S D$ & Min & Max & $p$-value \\
\hline \multirow{3}{*}{ Composite } & Star & 8.82 & 2.10 & 8.03 & 9.60 & \multirow{3}{*}{$0.001^{*}$} \\
\hline & Cross & 15.63 & 3.18 & 14.44 & 16.82 & \\
\hline & Slot & 4.61 & 2.30 & 3.74 & 5.47 & \\
\hline \multirow{3}{*}{ Amalgam } & Star & 6.40 & 2.22 & 5.57 & 7.23 & \multirow{3}{*}{0.180} \\
\hline & Cross & 7.28 & 3.82 & 5.85 & 8.71 & \\
\hline & Slot & 5.97 & 1.85 & 5.28 & 6.66 & \\
\hline
\end{tabular}

* statistically significant. 
Table 4. The adhesive remnant index (ARI) scores in 12 groups

\begin{tabular}{|c|c|c|c|c|c|c|c|c|}
\hline \multicolumn{3}{|c|}{ Group } & \multirow{2}{*}{$\begin{array}{c}\text { Score } 0 \\
n(\%)\end{array}$} & \multirow{2}{*}{$\begin{array}{c}\text { Score } 1 \\
n(\%)\end{array}$} & \multirow{2}{*}{$\begin{array}{c}\text { Score } 2 \\
n(\%)\end{array}$} & \multirow{2}{*}{$\begin{array}{c}\text { Score } 3 \\
n(\%)\end{array}$} & \multirow{2}{*}{$\begin{array}{c}\text { Fracture incidence in } \\
\text { the composite base } \\
n(\%)\end{array}$} & \multirow[b]{2}{*}{$p$-value } \\
\hline $\begin{array}{c}\text { restorative } \\
\text { material }\end{array}$ & $\begin{array}{c}\text { surface } \\
\text { preparation }\end{array}$ & $\begin{array}{c}\text { base } \\
\text { design }\end{array}$ & & & & & & \\
\hline \multirow{6}{*}{ Composite } & \multirow{3}{*}{ sandblasting } & Star & 0 & 0 & 0 & $\begin{array}{c}15 \\
(100.0)\end{array}$ & 0 & \multirow{12}{*}{$<0.001^{*}$} \\
\hline & & Cross & 0 & 0 & $\begin{array}{c}7 \\
(46.7)\end{array}$ & $\begin{array}{c}4 \\
(26.7)\end{array}$ & $\begin{array}{c}4 \\
(26.7)\end{array}$ & \\
\hline & & Slot & 0 & 0 & 0 & $\begin{array}{c}15 \\
(100.0)\end{array}$ & 0 & \\
\hline & \multirow{3}{*}{ laser } & Star & 0 & 0 & 0 & $\begin{array}{c}13 \\
(86.7)\end{array}$ & $\begin{array}{c}2 \\
(13.3)\end{array}$ & \\
\hline & & Cross & 0 & $\begin{array}{c}7 \\
(46.7)\end{array}$ & $\begin{array}{c}2 \\
(13.3)\end{array}$ & $\begin{array}{c}2 \\
(13.3)\end{array}$ & $\begin{array}{c}4 \\
(26.7)\end{array}$ & \\
\hline & & Slot & 0 & 0 & 0 & $\begin{array}{c}15 \\
(100.0)\end{array}$ & 0 & \\
\hline \multirow{6}{*}{ Amalgam } & \multirow{3}{*}{ sandblasting } & Star & $\begin{array}{c}9 \\
(60.0)\end{array}$ & 0 & 0 & $\begin{array}{c}6 \\
(40.0)\end{array}$ & 0 & \\
\hline & & Cross & $\begin{array}{c}15 \\
(100.0)\end{array}$ & 0 & 0 & 0 & 0 & \\
\hline & & Slot & $\begin{array}{c}13 \\
(86.7)\end{array}$ & $\begin{array}{c}2 \\
(13.3)\end{array}$ & 0 & 0 & 0 & \\
\hline & \multirow{3}{*}{ laser } & Star & $\begin{array}{c}15 \\
(100.0)\end{array}$ & 0 & 0 & 0 & 0 & \\
\hline & & Cross & $\begin{array}{c}15 \\
(100.0)\end{array}$ & 0 & 0 & 0 & 0 & \\
\hline & & Slot & $\begin{array}{c}13 \\
(86.7)\end{array}$ & $\begin{array}{c}2 \\
(13.3)\end{array}$ & 0 & 0 & 0 & \\
\hline
\end{tabular}

* statistically significant.

further studies are needed. Although a low bond strength is a disadvantage in the use of metal brackets, it can be a relative advantage when using ceramic brackets. One drawback of ceramic brackets is a high bond strength to the adhesives due to the chemical bond. As a result, the risk of damage to the enamel increases when the brackets are removed..$^{20}$

According to the results of this study, the mean shear bond strength value for the composite groups was 9.68 $\mathrm{MPa}$ and 6.55 $\mathrm{MPa}$ for the amalgam groups, and there was a significant difference between the 2 groups $(p<0.05)$. In fact, a lower bond strength was recorded on amalgam surfaces as compared to composite surfaces. The results obtained by Eslami Amirabadi et al. regarding the bond strength of stainless steel brackets bonded to porcelain and amalgam with Transbond XT and Assure ${ }^{\circledR}$ Universal Bond Resin are consistent with the current finding; the authors reported lower bond strength values on amalgam surfaces than on porcelain. ${ }^{21}$

Ebert et al. investigated the shear bond strength of ceramic and metal brackets bonded to composite, ceramic and amalgam restorative surfaces. ${ }^{22}$ The results showed that the shear bond strength of ceramic and metal brackets to amalgam surfaces was significantly lower than in other groups. ${ }^{22}$

In recent studies, the bond strength values on amalgam surfaces were significantly lower than on the enamel. ${ }^{4-10}$ However, considering a range of 5-8 $\mathrm{MPa}$ as the acceptable bond strength values in clinical conditions, ${ }^{8}$ the reported bond strength range in the amalgam and composite groups was acceptable in all our study cases except for 3 groups: composite + sandblasting + Slot; amalgam + laser + Star; and amalgam + laser + Cross.

According to the results of the present study, the mean shear bond strength value in the case of surface preparation with sandblasting was $8.84 \mathrm{MPa}$ and $7.40 \mathrm{MPa}$ for the laser group, and there was a significant difference between the 2 groups $(p<0.05)$. The mean shear bond strength values in the composite group with sandblasting and laser surface preparation were 9.22 $\mathrm{MPa}$ and 10.15 $\mathrm{MPa}$, respectively, which showed no significant difference; both groups provided adequate and sufficient bonds. The mean shear bond strength values in the amalgam group with sandblasting and laser surface preparation were $8.46 \mathrm{MPa}$ and $4.64 \mathrm{MPa}$, respectively, which showed a significant difference, and only sandblasting provided an adequate and sufficient bond. Therefore, both in the composite and amalgam groups, sandblasting surface preparation provided a sufficient and appropriate bond. The results of the research by Sperber et al., who studied the effects of different amalgam surface preparation methods, are consistent with the current finding; they reported higher shear bond strength values when preparing amalgam surfaces through sandblasting. ${ }^{10}$ 
A study by Espinar-Escalona et al. also confirms a higher shear bond strength for sandblasting surface preparation. ${ }^{23}$ Tayebi et al. evaluated different methods of composite surface preparation with sandblasting and rough diamond milling. ${ }^{16}$ Their results showed that the brackets bonded to composite surfaces had a higher shear bond strength in the sandblasted samples as compared to the surfaces prepared by means of a bur, although the difference was not statistically significant. ${ }^{16}$

Laser use in dentistry is currently increasing. ${ }^{24}$ Previous studies have shown that surface preparation with sandblasting is clinically acceptable and is recommended for bracket bonding on amalgam surfaces. In a similar study by Zaheer et al., the shear bond strength of amalgam samples was reported to be $16.30 \mathrm{MPa}$ with sandblasting surface preparation and 10.04 MPa with diamond bur preparation. ${ }^{25}$ Machado et al. studied the effects of different surface preparation methods, such as diamond bur or sandblasting, on amalgam samples that were packed inside composite cylinders. ${ }^{26}$ The sandblasted amalgam samples showed a higher bond strength than other groups. ${ }^{26}$ Another study was conducted to investigate the sandblasting and laser techniques for bonding metal brackets on amalgam surfaces. ${ }^{5}$ According to its results, sandblasting provided a higher bond strength in comparison with the control group, although there was no statistically significant difference. ${ }^{5}$

According to the results of this study, the average shear bond strength was $8.84 \mathrm{MPa}$ for the Star base design, 11.46 MPa for the Cross base design and 5.29 MPa for the Slot base design. Significant differences were observed when comparing the bond strength values in different groups of Star, Cross and Slot base designs.

The results obtained by Kukiattrakoon and Samruajbenjakul, who studied the shear bond strength of ceramic brackets with 3 base designs bonded to fluorapatite and aluminum ceramics, are consistent with the current finding; the researchers showed that there was a significant difference in shear bond strength for different bracket base designs. ${ }^{15}$

The results of Hudson et al.s study are consistent with ours. ${ }^{27}$ According to the authors, the different base designs of metal and ceramic brackets bonded to porcelain surfaces affected shear bond strength. ${ }^{27}$ Wang and Lu concluded that the bracket base design could be one of the factors influencing the shear bond strength of brackets. ${ }^{28}$

In the present study, in most of the amalgam groups $(88.8 \%)$, no adhesive remained on the restoration $(\mathrm{ARI}=0)$ and in most of the composite groups $(71.1 \%)$, all the adhesive remained on the restoration $(A R I=3)$. There was a significant difference in the ARI scores between the 2 groups $(p<0.001)$. The 0 score indicates that the fracture occurred at the adhesive-restoration interface, while the 3 score indicates that the fracture site was between the adhesive and the bracket base. The amount of the remaining adhesive is estimated in various studies based on ARI.
However, it is not possible to compare the results of different research in this field, as some studies have modified the ARI index and reported different results. Still, in most previous studies, the bond failure of the majority of samples was at the amalgam-adhesive interface $(\mathrm{ARI}=0),{ }^{29}$ which is consistent with our results.

A study by Tayebi et al. also provided similar results. ${ }^{16}$ The researchers showed that most composite samples had an index of 2 and 3, and some had a fracture in the composite base. ${ }^{16}$ This is due to the high strength of the bond between the adhesive and the prepared surface of the composite, which prevents a fracture at the composite-adhesive interface and causes a fracture at the adhesive-bracket base.

In the current study, the comparison of the ARI scores between the 3 groups of base design, including Star, Cross and Slot, showed that in the composite groups, the Star and Slot base designs had the highest amount of adhesive remaining on the restoration surface after debonding, and most cases of bond failure between the adhesive and the restoration occurred for the Cross base design. This base design causes more damage on the restoration surface than other designs. In the amalgam groups, the Cross base design showed more adhesive remnants on the restoration surface after debonding than other designs. In accordance with the current study, it was stated in previous studies that the type of base design had a great impact on ARI.

In a study by Ahangar Atashi et al., the comparison of the ARI scores between 2 groups with the anchor pylon base design and the mesh base design showed that in $20 \%$ of the mesh base design samples, bond failure occurred at the adhesive-enamel interface, and they also indicated the highest amount of adhesive remaining on the tooth surface after debonding. ${ }^{14}$ Bond failure between the adhesive and the enamel occurred most frequently for the anchor pylon base design. ${ }^{14}$ This base design leads to more stress being transferred to the enamel during debonding and causes more damage to the enamel surface than the mesh base design.

In a study by Ansari et al., the ARI scores in 4 groups of ceramic brackets and 1 group of metal brackets bonded to the enamel surface with different base designs were investigated. ${ }^{13}$ The groups with different base designs showed significant differences in terms of ARI, which is consistent with the present study.

In the current study, the shear bond strength test was performed using the looped ligature wire method, which is different from the usual blade method. The force from the testing machine was transferred to the upper jaw and the bracket by forming a ligature wire and connecting one end of the wire to the upper jaw and the other end to the bracket.

The crosshead speed in the current study was $1 \mathrm{~mm} / \mathrm{min}$. A crosshead speed of $0.1-10 \mathrm{~mm} / \mathrm{min}$ have been used for shear bond strength testing; however, 
these values do not correspond to the values in the clinical oral environment, because the speed of mastication is in range of $81-100 \mathrm{~mm} / \mathrm{s}$ or $4,860-6,000 \mathrm{~mm} / \mathrm{min}$ with a frequency of $1.03-12 \mathrm{~Hz} .^{30}$

Storage media in previous investigations were water or normal saline. ${ }^{4}$ The storage temperature was $37^{\circ} \mathrm{C}$ and the shelf life also varied from $24 \mathrm{~h}$ to 10 weeks. In the present study, the samples were stored in an incubator at $37^{\circ} \mathrm{C}$ and $95 \%$ humidity for 1 week, and then subjected to 10,000 thermal cycles for artificial aging. They were soaked in $5^{\circ} \mathrm{C}$ cold water for $20 \mathrm{~s}$ and placed in $55^{\circ} \mathrm{C}$ warm water for $20 \mathrm{~s}$ in each cycle. ${ }^{10}$ This may be one of the reasons for differences in the results of various studies. Undoubtedly, simulating oral conditions will never be fully possible in vitro.

\section{Conclusions}

The results of the study are limited by the laboratory conditions and further studies should be performed in a clinical setting. Within the limitations of this in vitro study, all surface treatment methods and different bracket base designs were able to provide sufficient shear bond strength on composite and amalgam surfaces. Considering bond strength in all groups, surface treatment by means of sandblasting is recommended in clinical practice due to its availability, lower cost and fewer injuries it causes while working. In terms of bracket base design, according to ARI and the bond strength values, the use of each of the 3 base designs in the amalgam group and the use of the Star base design in the composite group are suitable.

\section{ORCID iDs}

Maryam Shirazi (1) https://orcid.org/0000-0001-7377-4435 Monirsadat Mirzadeh (10) https://orcid.org/0000-0001-7479-2395 Malek Modirrousta (1) https://orcid.org/0000-0002-5388-3420 Sepideh Arab (10) https://orcid.org/0000-0001-6936-875X

\section{References}

1. Wongsamut W, Satrawaha S, Wayakanon K. Surface modification for bonding between amalgam and orthodontic brackets. J Orthod Sci. 2017;6(4):129-135. doi:10.4103/jos.JOS_25_17

2. Boruziniat A, Khazaei $Y$, Motaghi S, Moghaddas M. Evaluation of bond strength of orthodontic brackets without enamel etching. J Clin Exp Dent. 2015;7(4):e519-e523. doi:10.4317/jced.52253

3. Elnafar AAS, Alam MK, Hasan R. The impact of surface preparation on shear bond strength of metallic orthodontic brackets bonded with a resin-modified glass ionomer cement. J Orthod. 2014;41(3):201-207. doi:10.1179/1465313314Y.0000000097

4. Büyükyilmaz T, Zachrisson BU. Improved orthodontic bonding to silver amalgam. Part 2. Lathe-cut, admixed, and spherical amalgams with different intermediate resins. Angle Orthod. 1998;68(4):337-344. doi:10.1043/0003-3219(1998)068<0337:IOBTSA $>2.3 . \mathrm{CO} ; 2$

5. Oskoee PA, Kachoei M, Rikhtegaran S, Fathalizadeh F, Navimipour EJ. Effect of surface treatment with sandblasting and Er,Cr:YSGG laser on bonding of stainless steel orthodontic brackets to silver amalgam. Med Oral Patol Oral Cir Bucal. 2012;17(2):e292-e296. doi:10.4317/medoral.17473
6. Ülker M, Malkoç S, Ülker HE, Yalçin M, Malkoç M. Orthodontic bonding to high-copper amalgam with different adhesive cements. J Restorat Dent. 2016;4(1):7-10. doi:10.4103/2321-4619.176015

7. Demirtas HK, Akin M, lleri Z, Basciftci FA. Shear-bond-strength of orthodontic brackets to aged nano-hybrid composite-resin surfaces using different surface preparation. Dent Mater J. 2015;34(1):86-90. doi:10.4012/dmj.2014-115

8. Zachrisson BU, Büyükyilmaz T, Zachrisson YO. Improving orthodontic bonding to silver amalgam. Angle Orthod. 1995;65(1):35-42. doi:10.1043/0003-3219(1995)065<0035:IOBTSA >2.0.CO;2

9. Usumez $A$, Aykent $F$. Bond strengths of porcelain laminate veneers to tooth surfaces prepared with acid and Er,Cr:YSGG laser etching. J Prosthet Dent. 2003;90(1):24-30. doi:10.1016/s0022-3913(03)00235-x

10. Sperber RL, Watson PA, Rossouw PE, Sectakof PA. Adhesion of bonded orthodontic attachments to dental amalgam: In vitro study. Am J Orthod Dentofacial Orthop. 1999;116(5):506-513. doi:10.1016/s0889-5406(99)70180-0

11. Karamouzos A, Athanasiou AE, Papadopoulos MA. Clinical characteristics and properties of ceramic brackets: A comprehensive review. Am J Orthod Dentofacial Orthop. 1997;112(1):34-40. doi:10.1016/s0889-5406(97)70271-3

12. Sturdevant CM. Biomaterials. In: Roberson TM, Heymann H, Swift EJ, eds. Sturdevant's Art \& Science of Operative Dentistry. $4^{\text {th }}$ ed. St Louis, USA: Mosby; 2002:140-186.

13. Ansari MY, Agarwal DK, Gupta A, Bhattacharya P, Ansar J, Bhandari R. Shear bond strength of ceramic brackets with different base designs: Comparative in-vitro study. J Clin Diagn Res. 2016;10(11):ZC64-ZC68. doi:10.7860/JCDR/2016/20624.8910

14. Ahangar Atashi MH, Sadr Haghighi AH, Nastarin $P$, Ahangar Atashi S. Variations in enamel damage after debonding of two different bracket base designs: An in vitro study. J Dent Res Dent Clin Dent Prospects. 2018;12(1):56-62. doi:10.15171/joddd.2018.009

15. Kukiattrakoon B, Samruajbenjakul B. Shear bond strength of ceramic brackets with various base designs bonded to aluminous and fluorapatite ceramics. Eur J Orthod. 2010;32(1):87-93. doi:10.1093/ejo/cjp055

16. Tayebi A, Fallahzadeh F, Morsaghian M. Shear bond strength of orthodontic metal brackets to aged composite using three primers. J Clin Exp Dent. 2017;9(6):e749-e755. doi:10.4317/jced.53731

17. Yetkiner $E$, Özcan $M$. Adhesive strength of metal brackets on existing composite, amalgam and restoration-enamel complex following air-abrasion protocols. Int J Adhes Adhes. 2014;54:200-205. doi:10.1016/J.IJADHADH.2014.06.012

18. Chaconas SJ, Caputo AA, Niu GS. Bond strength of ceramic brackets with various bonding systems. Angle Orthod. 1991;61(1):35-42. doi:10.1043/0003-3219(1991)061<0035:BSOCBW>2.0.CO;2

19. Elekdag-Turk $S$, Turk $T$, Isci $D$, Ozkalayci N. Thermocycling effects on shear bond strength of a self-etching primer. Angle Orthod. 2008;78(2):351-356. doi:10.2319/122906-537.1

20. Mehmeti B, Azizi B, Kelmendi J, Iljazi-Shahiqi D, Alar Ž, AnićMilošević $S$. Shear bond strength of orthodontic brackets bonded to zirconium crowns. Acta Stomatol Croat. 2017;51(2):99-105. doi:10.15644/asc51/2/2

21. Eslami Amirabadi GR, Shirazi M, Shirazi Z. Micro-shear bond strength of Transbond XT and Assure Universal Bonding Resin to stainless steel brackets, amalgam and porcelain. J Islam Dent Assoc Iran. 2015;27(1):1-5.

22. Ebert T, Elsner L, Hirschfelder U, Hanke S. Shear bond strength of brackets on restorative materials: Comparison on various dental restorative materials using the universal primer Monobond ${ }^{\circledR}$ Plus. J Orofac Orthop. 2016;77(2):73-84. doi:10.1007/s00056-016-0011-y

23. Espinar-Escalona E, Barrera-Mora JM, Llamas-Carreras JM, SolanoReina E, Rodríguez D, Gil FJ. Improvement in adhesion of the brackets to the tooth by sandblasting treatment. J Mater Sci Mater Med. 2012;23(2):605-611. doi:10.1007/s10856-011-4509-y

24. Fabianelli A, Pollington S, Papacchini F, et al. The effect of different surface treatments on bond strength between leucite reinforced feldspathic ceramic and composite resin. J Dent. 2010;38(1):39-43. doi:10.1016/j.jdent.2009.08.010

25. Zaheer U, Zaheer N, Shahbaz M. Shear bond strength of brackets on bonding to treated amalgam surfaces. Pak Oral Dent J. 2018;38(2):207-210. 
26. Machado C, Sanchez E, Alapati S, Seghi R, Johnston W. Shear bond strength of the amalgam-resin composite interface. Oper Dent. 2007;32(4):341-346. doi:10.2341/06-100

27. Hudson AP, Grobler SR, Harris AMP. Orthodontic molar brackets: The effect of three different base designs on shear bond strength. Int J Biomed Sci. 2011;7(1):27-34.

28. Wang WN, Lu TC. Bond strength with various etching times on young permanent teeth. Am J Orthod Dentofacial Orthop. 1991;100(1):72-79. doi:10.1016/0889-5406(91)70052-X

29. Al Jabbari YS, Al Taweel SM, Al Rifaiy M, Alqahtani MQ, Koutsoukis T, Zinelis S. Effects of surface treatment and artificial aging on the shear bond strength of orthodontic brackets bonded to four different provisional restorations. Angle Orthod. 2014;84(4):649-655. doi:10.2319/090313-649.1

30. Buschang PH, Hayasaki $H$, Throckmorton GS. Quantification of human chewing-cycle kinematics. Arch Oral Biol. 2000;45(6):461-474. doi:10.1016/s0003-9969(00)00015-7 